\title{
Comment on "Mapping the dayside ionosphere to the magnetosphere according to particle precipitation characteristics" by Newell and Meng
}

Article

Published Version

Lockwood, M. and Smith, M. F. (1993) Comment on "Mapping the dayside ionosphere to the magnetosphere according to particle precipitation characteristics" by Newell and Meng. Geophysical Research Letters, 20 (16). pp. 1739-1740. ISSN 00948276 doi: https://doi.org/10.1029/93GL01967 Available at https://centaur.reading.ac.uk/38820/

It is advisable to refer to the publisher's version if you intend to cite from the work. See Guidance on citing.

Published version at: http://dx.doi.org/10.1029/93GL01967

To link to this article DOI: http://dx.doi.org/10.1029/93GL01967

All outputs in CentAUR are protected by Intellectual Property Rights law, including copyright law. Copyright and IPR is retained by the creators or other copyright holders. Terms and conditions for use of this material are defined in the End User Agreement. 


\section{CentAUR}

Central Archive at the University of Reading

Reading's research outputs online 


\title{
COMMENT ON "MAPPING THE DAYSIDE IONOSPHERE TO THE MAGNETOSPHERE ACCORDING TO PARTICLE PRECIPITATION CHARACTERISTICS" BY NEWELL AND MENG
}

\author{
M. Lockwood \\ Space Science Department, Rutherford Appleton Laboratory \\ and M. F. Smith \\ Laboratory for Extraterrestrial Physics, Goddard Space Flight Center
}

In their paper, Newell and Meng (1992) present some maps of the occurrence probability of various classifications of particle precipitation, as seen in the dayside topside ionosphere. They also state that these plots are maps of the magnetospheric regions and it is this concept with which we disagree. To illustrate our point we restrict this comment to the magnetosheath-like populations termed 'LLBL', 'cusp' and 'mantle', but similar arguments would apply to the CPS and BPS populations which arise within the magnetosphere.

The concept of plasma populations arising from a structured magnetosphere mapping to the ionosphere only applies to a stagnant magnetosphere. The populations mapped in the ionosphere by Newell and Meng all appear in regions where there is convection and hence must have all been subject to dispersion by the convection of the field lines as they travel along the field lines on which they are frozen. Two particles of different energy (but the same mass and pitch angle), which are seen simultaneously at one point in the ionosphere have different flight times and hence cannot have arisen from the same point in the magnetosphere (unless the field line does not move - i.e. a stagnant magnetosphere) (Lockwood and Smith, 1992; Onsager et al., 1993). Hence the population seen in the ionosphere is an ensemble, with particles coming from a variety of magnetospheric locations.

The ionospheric population could be identical to that in the magnetosphere in the presence of convection, only if there is no spatial structure in the magnetosphere - in which case there would be none in the ionosphere either. However, Newell and Meng define boundaries between regions of ionospheric precipitation and identify them with boundaries between source populations in the magnetosphere. Because of convection, this is incorrect. Hence although it is useful to define populations in the ionosphere, it is misleading to name them 'LLBL', 'cusp' and 'mantle' because this implies that each population comes from the magnetospheric region bearing the same name. This is not the case.

To illustrate the point, let us consider the 'cusp' precipitation. The dispersion of cusp ions by convection is well known and was first described by Rosenbauer et al (1975). Observations show that the cusp is always electrically neutral (Burch, 1985). Due to the higher mass and momentum of the cusp ions (relative to the electrons), their dispersion is well explained by adiabatic scatter-free motion. Hence it must be the electron gas behaviour that is modified in order to maintain neutrality. As a result, the electron characteristics are heavily influenced by the ions and we therefore restrict our attention to the latter. Newell and Meng

Copyright 1993 by the American Geophysical Union.

Paper number 93GL01967

0094-8534/93/93GL-01967\$03.00 employ a definition which results in a spread of ion energies between, typically, $E_{1}=200 \mathrm{eV}$ and $E_{2}=2 \mathrm{keV}$ in the region designated 'cusp' (see examples given by Newell and Meng, 1988). These protons are observed at very close to zero pitch angle, so they have field-aligned speeds of $v_{1}=200 \mathrm{~km} \mathrm{~s}^{-1}$ and $v_{2}=620 \mathrm{~km} \mathrm{~s}^{-1}$. If the lower and upper energy ions are injected from the magnetosheath at distances from the satellite along the field line of $d_{1}$ and $d_{2}$, respectively, they are both observed in the ionosphere at time $t_{0}$ but were injected at times $\left(t_{0}-d_{1} / v_{1}\right)$ and $\left(t_{0}-d_{2} / v_{2}\right)$. Between these two times the injection point has convected a distance $\Delta$ given by

$$
\Delta=V_{F}\left[\left(d_{1} / v_{1}\right)-\left(d_{2} / v_{2}\right)\right]
$$

where $V_{F}$ is the field line convection velocity along the magnetopause which is taken to be constant for simplicity. The ion trajectories for observation at a point $\mathrm{C}$ in the cusp are shown in figure 1. Both theory and observation (Smith and Rodgers, 1991, and references therein) show that the minimum injected energy corresponds to speed $v_{1}=V_{F}$ at the dayside magnetopause and $V_{F}$ can easily exceed $200 \mathrm{~km} \mathrm{~s}^{-1}$ (e.g. Sonnerup et al., 1990). Later, as it passes through the magnetic cusp, an open field line will be moving at roughly the magnetosheath flow speed of about $240 \mathrm{kms}^{-1}$ for a solar

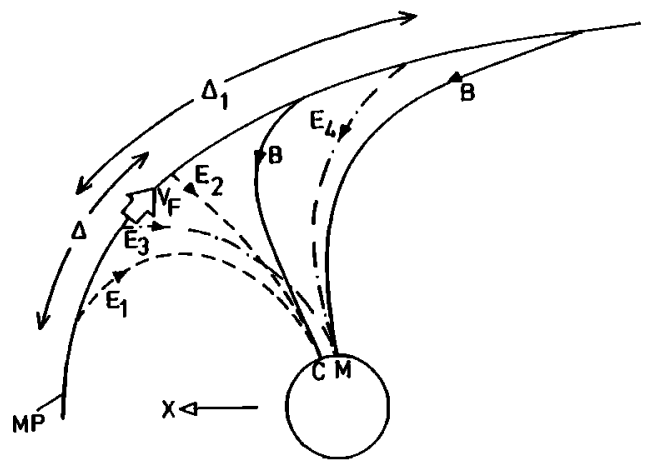

Fig. 1. Some trajectories of magnetosheath ions in the magnetosphere after injection across the magnetopause (MP). The point $\mathrm{C}$ is where a low-altitude satellite sees a 'cusp' population, whereas it sees a 'mantle' one at $M$. The cusp ions with maximum energy $\left(\mathrm{E}_{2}\right)$ and minimum energy $\left(E_{1}\right)$ have followed the trajectories shown by the dashed lines as they precipitate down the convecting field lines (only for a stagnant magnetosphere are these trajectories field-aligned). The mantle ions of maximum and minimum energy $\left(E_{4}\right.$ and $E_{3}$ respectively) have followed the dot-dash lines. The solid lines show the magnetic field lines through $C$ and $M$. The field line moves at speed $V_{F}$ at the magnetopause, and $\Delta$ and $\Delta_{\mathfrak{l}}$ give the ranges of injection locations for ions seen at $C$ and $M$. (c.f. Figure 1 and text of Onsager et al., 1993). 
wind speed $V_{s w}$ of $400 \mathrm{~km} \mathrm{~s}^{-1}$. Antisunward of the cusp $V_{F}$ will increase toward $V_{s w}$. We derive a minimum value for $\Delta$ by taking a minimum distance from the magnetopause to the satellite of $d_{1}=d_{2}=7 R_{E}$ and a minimum $V_{F}$ of $200 \mathrm{~km} \mathrm{~s}^{-1}$ : from equation (1), $\Delta \approx 5 R_{\mathrm{E}}$. However, this is a gross underestimate because as the field line evolves over the magnetopause, the distance to the ionosphere shortens $\left(d_{1}>\right.$ $d_{2}$ ). The shape of the dayside field means that $d_{1} \approx f\left(d_{2}+\Delta\right)$, where $f$ is a geometrical factor of order, but less than, unity (the field line runs along the dayside magnetopause to close to the magnetic cusp and then to the ionosphere). Hence

$\Delta=\mathrm{d}_{2}\left[\left(f / V_{1}\right)-\left(1 / V_{2}\right)\right] /\left[\left(1 / V_{F}\right)-\left(f / V_{1}\right)\right]$

which yields $\Delta=17 R_{E}$ for $f=0.8$ and $d_{2}=7 R_{E}$. This demonstrates the broad spread of origins of ions seen simultaneously in the ionosphere: essentially, the lowest energy ions originated from the subsolar magnetopause, but the higher energies observed came from the magnetic cusp.

The range of locations from which ions originate to make up the 'mantle' population in the ionosphere is even greater. For example $E_{3}=50 \mathrm{eV}, E_{4}=1 \mathrm{keV}$ is a typical range in this region. Even using equation (1) with $d_{1}=d_{2}=7 R_{E}$ we derive a minimum value for $\Delta_{\mathrm{l}}$ of $11 \mathrm{R}_{\mathrm{E}}$. Note that this too is a gross underestimate because both the $d_{2}$ and $V_{F}$ values are too small. Any $50 \mathrm{eV}$ 'mantle' ions close to the cuspmantle boundary are injected from a point several tens of $R_{E}$ sunward of a $1 \mathrm{keV}$ cusp ions adjacent to that boundary - i.e. the source regions of cusp and mantle particles overlap, as demonstrated in figure 1 . Hence the field line on which 'cusp'-'mantle' ionospheric boundary lies does not separate two populations in the magnetosphere. Similar arguments apply to the LLBL-cusp and mantle-polar rain boundaries.

From the above we conclude that, because of the range of times of flight in a convecting magnetosphere (i.e. dispersion effects), populations in the ionosphere originate from a wide variety of magnetospheric locations and cannot be mapped to magnetospheric regions. Hence the use of the terms 'LLBL', 'cusp' and 'mantle', although widespread, is inherently misleading. Instead, the precipitation at the ionospheric part of a newly-opened field line should be considered as evolving from one classification to another: LLBL to cusp to mantle to polar rain (Cowley et al., 1991). In those (steadystate) cases mentioned by Newell and Meng, where the mantle is a 'cusp plume', this kind of evolution self-evident. Hence the particle characteristics in the ionosphere depend upon the time elapsed since that field line was reconnected.

The time-of-flight considerations mean that only higher energies have arrived in the ionospheric 'LLBL' region, and hence the average energy will be higher but the average density will be lower than for the cusp. In their original paper defining differences between the LLBL and the cusp, Newell and Meng (1988) dismiss this possibility because both populations are seen in one pass, with an apparent boundary between them. Step-like changes in any plasma flux value do undoubtedly occur at the boundary between any two regions. However, the only way that such steps can be interpreted as a spatial boundary extending up field lines into the magnetosphere is if there is no convection across it i.e no poleward flow from the LLBL to cusp to mantle to polar cap. Lockwood and Smith (1992) have recently shown that jumps in the particle characteristics are explained by temporal variations in the rate of magnetic reconnection and Lockwood et al. (1993) have shown that the precipitation on newly-opened field lines can evolve from 'LLBL' to 'cusp' to 'mantle' with time elapsed since reconnection. However, we also note that the above does not preclude a second type of LLBL on closed field lines.

In summary, because of convection, any one population of particles seen at low-altitudes will have originated from a wide variety of locations and particle characteristics cannot be mapped back to those in the magnetosphere without detailed knowledge of both the convection and magnetic field. The population seen at the foot of any one open field line will evolve continuously through the classifications 'LLBL', 'cusp', 'cleft' and 'polar rain'. Step-like boundaries between the regions will arise from non-steady-state conditions and cannot be envisaged as steady-state magnetospheric boundaries between two plasma populations.

\section{References}

Burch, J. L., Quasi-neutrality in the polar cusp, Geophys. Res. Lett., 12, 469, 1985.

Cowley, S.W.H.et al., The ionospheric signature of flux transfer events in "CLUSTER - dayside polar cusp", ESA SP-330, ed. C.I Barron. European Space Agency Publ., Nordvijk, The Netherlands, pp 105-112, 1991.

Lockwood, M., et al., Ionospheric signatures of pulsed magnetic reconnection at the Earth's magnetopause, Nature, 361 (641I), 424, 1993.

Lockwood, M. and M.F. Smith, The variation of reconnection rate at the dayside magnetopause and cusp ion precipitation, J. Geophys. Res., 97, 14841, 1992.

Newell, P. T. and C. I. Meng, The cusp and the cleft/ LLBL: Low altitude identification and statistical local time variation, J. Geophys. Res., 93, 14549, 1988

Newell, P.T. and C.-I. Meng, Mapping the dayside ionosphere to the magnetosphere according to particle precipitation characteristics, Geophys.Res_Lett., 19, 609, 1992

Onsager, T.G., et al., Model of magnetosheath plasma in the magnetosphere: cusp and mantle particles at low altitudes, Geophys. Res. Lett., 20, 47, 1993.

Rosenbauer, H., et al., HEOS-2 plasma observations in distant polar magnetosphere: the plasma mantle, $J$. Geophys. Res., 80, 2723, 1975.

Smith, M.F. and Rodgers, D.J., Ion distributions at the dayside magnetopause, J.Geophys.Res., 95, 11617,1991

Sonnerup, B.U.O., et al., The magnetopause for large magnetic shear: analysis of convection electric fields from AMPTE/IRM, J. Geophys. Res., 95, 10054, 1990

M. Lockwood, Rutherford Appleton Laboratory, Chilton, Didcot, OX11 0QX, U.K.

M.F. Smith, Laboratory for Extraterrestrial Physics,

Goddard Space Flight Center, Greenbelt, MD, U.S.A.

(Received February 18, 1993;

revised April 22, 1993;

accepted June 14, 1993.) 